\title{
Emparelhamento por Identidade e TEA: Efeito de Pares de Estímulos Idênticos como Consequência de Pareamentos Corretos
}

\author{
Kelvis Rodrigo Sampaio da Cruz ${ }^{1}$ \\ Orcid.org/0000-0002-5910-5427 \\ Raquel Maria de Melo ${ }^{2, *}$ \\ Orcid.org/0000-0003-2414-0633 \\ ${ }^{I}$ Faculdade de Ensino Superior do Piauí, Teresina, PI, Brasil \\ ${ }^{2}$ Universidade de Brasília, Brasília, DF, Brasil
}

\section{Resumo}

Em uma replicação parcial do estudo de Gomes (2011), foi avaliado o efeito da apresentação de pares de estímulos idênticos após pareamentos corretos em tarefas de emparelhamento Típico e Multimodelo. Participaram do estudo 24 indivíduos com Transtorno do Espectro Autista (TEA), divididos em duas condições, com três blocos de tentativas cada: Típico, Multimodelo e os dois tipos misturados. A ordem de exposição foi contrabalanceada entre os participantes de uma mesma condição. Os dois primeiros blocos continham tentativas de treino e de teste e o terceiro bloco apenas tentativas de teste. $\mathrm{Na}$ Condição 1, a cada pareamento correto o estímulo modelo era removido da tela e na Condição 2 era apresentado um estímulo composto com dois elementos iguais. Os resultados mostraram que, independentemente da condição e da ordem de exposição as tarefas, as porcentagens de acerto foram iguais ou maiores nas tentativas de teste de Emparelhamento Típico. Na Condição 2, os escores foram superiores aos da Condição 1. Para indivíduos com TEA, o controle pela relação de identidade pode ser afetado pela organização visual das tarefas e por outras variáveis de procedimento tais como as consequências para os pareamentos, a topografia da resposta (clicar ou arrastar), e o critério nos treinos.

Palavras-chave: Emparelhamento de identidade, emparelhamento multimodelo, relações condicionais, autismo.

* Endereço para correspondência: Universidade de Brasília, Departamento de Processos Psicológicos Básicos/ Instituto de Psicologia, Campus Universitário Darci Ribeiro, Brasília, DF, Brasil 70.910-900. Fone: (61) 3107 2844; Fax: (61) 3273-0203. E-mail: melo.rm@gmail.com

O estudo é parte da dissertação de mestrado do primeiro autor realizado no Programa de Pós-Graduação em Ciências do Comportamento da Universidade de Brasília, sob a orientação da segunda autora, e contou com a co-orientação da Profa. Dra. Camila Graciella Santos Gomes. O desenvolvimento do estudo foi apoiado pelo Instituto Nacional de Ciência e Tecnologia sobre Comportamento, Cognição e Ensino, financiado pelo Conselho Nacional de Desenvolvimento Científico e Tecnológico - CNPq (Processo \#573972/2008-7) e pela Fundação de Amparo à Pesquisa do Estado de São Paulo - FAPESP (Processo \# 2008/57705-8). 


\title{
Identity Matching-To-Sample and ASD: Effect of Pairs of Identical Stimuli as a Consequence for Correct Matching
}

\begin{abstract}
In a replication of a study by Gomes (2011), the effect of the presentation of pairs of identical stimuli after correct matching was assessed in Typical and Multi-sample matching-to-sample tasks. Twenty-four individuals with Autism Spectrum Disorder (ASD) were distributed in two conditions, each with three blocks of trials: Typical, Multi-sample and a mix of both trial types. The order of exposure was counterbalanced across participants within a condition. The first two blocks of trials consisted of both training and testing trials; the third block consisted only of test trials. In Condition 1, the sample stimulus was removed from the screen upon correct matching; in Condition 2 correct matching was followed by presentation of a compound stimulus with two equal elements. Results showed that, regardless of experimental condition and order of exposure to matching-to-sample tasks, percentage of correct responses was higher in Typical Matching test trials. In Condition 2, scores were greater than in Condition 1. For individuals with ASD, control by the relation of identity may be affected by the visual organization of the tasks and by other procedural variables such as the consequences for matching responses, response topography (clicking or dragging) and the training criterion.
\end{abstract}

Keywords: Identity matching, multi-sample matching, conditional relations, autism.

\section{Igualación de Identidad y TEA: Efecto de Pares de Estímulos Idénticos como Consecuencias para la Igualación Correcta}

\section{Resumen}

En una réplica parcial del estudio de Gomes (2011), se evaluó el efecto de la presentación de parejas de estímulos idénticos después de emparejamientos correctos en tareas de igualación Típica y Multimuestra. Participaron del estudio 24 individuos con Trastorno del Espectro Autista (TEA), divididos en dos condiciones con tres bloques de ensayos: Típico, Multimuestra y los dos tipos mezclados. Los dos primeros bloques tenían ensayos de entrenamiento y prueba, mientras que el tercer bloque tuvo solamente ensayos de prueba. En la Condición 1, el estímulo muestra era retirado de la pantalla después de cada respuesta correcta mientras que, en la Condición 2, un estímulo compuesto con dos elementos iguales era presentado. Independiente de la condición y del orden de exposición a las tareas, el porcentaje de aciertos fue igual o mayor en los ensayos de prueba con emparejamiento Típico. Asimismo, los puntajes fueron iguales o más altos en la Condición 2. Para individuos con TEA, el control por las relaciones de identidad puede ser afectado por la organización visual de la tarea y por otras variables de procedimiento, tales como las consecuencias para las respuestas, la topografía de la respuesta y los criterios de entrenamiento.

Palablas clave: Igualación a la muestra, igualación multi-muestra, relaciones condicionales, autismo.

Dube (1996) descreveu um programa de ensino de habilidades de discriminação para indivíduos com dificuldade de aprendizagem, deficiência intelectual e/ou com TEA. Tal programa estabelece uma rota, ou sequência, que inicia com o ensino de habilidades discriminativas mais simples. Tarefas cada vez mais complexas são inseridas de acordo com o progresso do aprendiz. Assim, o ensino de relações de identidade e a verificação de identidade generalizada (identificar quaisquer estímulos iguais e não apenas os que foram explicitamente ensinados) devem preceder o ensino de relações arbitrárias, tais como relações entre palavras ditadas e figuras e entre palavras ditadas e palavras impressas (Dube, 1996; Kelly, Green, \& Sidman, 1998). 
Verifica-se, na literatura, relatos sobre a dificuldade no ensino de relações condicionais para indivíduos com TEA (Eikeseth \& Smith, 1992; Kelly et al., 1998; Williams, Pérez-Gonzáles, \& Queiroz, 2005). Em geral, tais participantes necessitam de um número maior de tentativas de treino para aprender relações condicionais em comparação com indivíduos com desenvolvimento típico.

O procedimento comumente utilizado para o ensino de relações condicionais é o emparelhamento ao modelo. No caso de relações de identidade, diante de cada estímulo modelo, respostas de seleção do estímulo que apresenta características físicas similares ao modelo, dentre as alternativas disponíveis, ou estímulos de comparação, são correlacionadas com reforço (Albuquerque \& Melo 2005; Stromer \& Stromer, 1989). A organização visual das tarefas de emparelhamento ao modelo têm sido apontada como uma variável que afeta o desempenho dos participantes (Gomes, 2011; Gomes \& de Souza, 2008; Mesibov, Schopler, \& Hearsey, 1994).

Gomes e de Souza (2008) compararam o desempenho de 20 indivíduos com TEA (leve/moderado e grave) em dois procedimentos de emparelhamento de identidade que diferiam quanto a organização visual da apresentação dos estímulos. As tentativas de cada tarefa foram apresentadas em um fichário composto por divisórias (páginas), nas quais eram inseridos cartões com os estímulos (figuras familiares, palavras, sequências de letras ou símbolos abstratos). Em cada tentativa da tarefa de Emparelhamento Típico, a página da esquerda continha um sinalizador (palito de madeira com velcro) e na página à direita eram apresentados o estímulo modelo fixo, na parte superior, e três estímulos de comparação fixos na parte inferior. A resposta de seleção do participante consistia em pegar o palito e colocá-lo sobre o velcro abaixo de um dos estímulos de comparação. Na tarefa de Emparelhamento Multimodelo, em cada tentativa, eram apresentados na página da esquerda três estímulos de comparação móveis e, na página da direita, três estímulos modelo fixos. O participante deveria pegar, um a um, os três estímulos da página da esquerda e colocá-los sobre o estímulo igual localizado na página da direita. A tentativa era finalizada quando os três emparelhamentos eram efetuados, indicado pela página da esquerda vazia. $\mathrm{O}$ procedimento era composto por três blocos de tentativas: Emparelhamento Multimodelo, Emparelhamento Típico e, por último, os dois tipos de tarefas misturadas. Com um mínimo de treino, foi verificado que a porcentagem média de acerto nas tentativas de Emparelhamento Multimodelo foi significativamente maior do que nas tentativas de Emparelhamento Típico. Entretanto, ocorreu variabilidade nos desempenhos dos participantes nas duas tarefas, principalmente para os participantes não verbais.

No estudo de Gomes e de Souza (2008), a ordem de exposição às tarefas pode ter favorecido o melhor desempenho nas tentativas de Emparelhamento Multimodelo (sempre a primeira tarefa). Para controlar essa variável, Gomes (2011; Experimento 1) realizou uma replicação com 40 indivíduos com TEA e 40 com deficiência intelectual. Metade dos participantes com TEA e metade com deficiência intelectual realizaram as tarefas em papel e os demais participantes realizaram as tarefas no computador. A ordem de exposição aos dois tipos de emparelhamentos foi balanceada. As tarefas apresentavam organização visual similar as do estudo de Gomes e Souza (2008). Entretanto, no computador era requerido no Emparelhamento Típico a resposta de clicar em um dos estímulos de comparação correspondente ao modelo. No Emparelhamento Multimodelo, o participante deveria clicar em um dos estímulos de comparação, na parte esquerda da tela, arrastá-lo e colocá-lo sobre o estímulo modelo correspondente (à direita). Nos dois tipos de emparelhamento, era permitida mudança da resposta de escolha, ou do estímulo colocado sobre o modelo, antes de clicar no botão de finalização da tentativa. Independente da ordem de exposição, para a maioria dos participantes foi observada porcentagem de acerto mais alta nas tentativas de Emparelhamento Multimodelo, tanto nas tarefas realizadas em papel como no computador, o que replica os resultados do estudo anterior.

Gomes (2011) sugeriu que a maior porcentagem de acerto nas tentativas de Emparelha- 
mento Multimodelo poderia estar relacionada com a organização visual dos estímulos modelo e de comparação e com a topografia da resposta requerida. Na tarefa de Emparelhamento Multimodelo, o participante deveria colocar cada um dos três estímulos de comparação sobre o seu respectivo modelo. Esse contexto pode ter favorecido a resposta de olhar tanto para os estímulos de comparação quanto para os estímulos modelo, aumentando a probabilidade de acerto e o controle pela relação de igualdade. Em cada tentativa, quando o participante colocava um estímulo sobre o outro (ou arrastava no computador), mudava a organização visual da tarefa, pois diminuía o número de estímulos de um dos lados. Além disso, a redução do número de estímulos de comparação resultava em aumento da probabilidade de acerto, de 33,33\% com três estímulos para $100 \%$, após o segundo emparelhamento, quando estava disponível apenas uma alternativa de escolha.

A topografia da resposta em tarefas no computador também tem sido apontada como uma variável relevante para o estabelecimento de controle pela relação de identidade. Shimizu, Twyman e Yamamoto (2003) avaliaram o desempenho de crianças, com atraso no desenvolvimento, em tarefas de identidade com Emparelhamento Típico com duas respostas distintas. $\mathrm{Na}$ Condição Clicar, o participante deveria clicar sobre o estímulo de comparação correspondente ao modelo e na Condição Arrastar, era necessário clicar sobre o modelo e arrastá-lo, com o uso mouse, até posicioná-lo abaixo do estímulo de comparação correspondente. Foi verificada porcentagem de acerto maior na Condição Arrastar. Em outro estudo, com três crianças com deficiência intelectual, de Freitas (2012; Experimento 2) utilizou um procedimento suplementar para ensinar relações de identidade entre palavras com três letras. Era ensinada uma sequência de respostas: olhar para o modelo, clicar sobre um dos estímulos de comparação, arrastá-lo até o modelo e soltá-lo. Ao longo das etapas de treino, o estímulo de comparação deveria ser movimentado por distância cada vez menores até que não fosse mais necessário arrastá-lo, mas apenas clicar. Com a utilização desse procedimento, todos os participantes apresentaram porcentagem de acerto alta (superior a $80 \%$ ) e mantiveram desempenhos similares nos treinos de novas relações de identidade.

A investigação de variáveis de procedimentos que afetam a aprendizagem de relações de identidade por indivíduos com TEA é relevante, pois tais relações são a base para a aquisição de relações arbitrárias (Dube, 1996) que estão envolvidas em comportamentos simbólicos tais como linguagem e conceitos (de Rose, 1993; de Rose \& Bortoloti, 2007; Smith, 2001). Comportamentos simbólicos envolvem respostas sob controle de relações arbitrárias entre estímulos e, portanto, comprometimentos na aprendizagem de relações de identidade e arbitrárias podem implicar em habilidades disfuncionais de comunicação, atraso no desenvolvimento da linguagem, fracasso em competências básicas em leitura, escrita e matemática, dentre outros (de Sousa, Cortez, Aggio, \& de Rose, 2012).

Nesse sentido, características do procedimento de Emparelhamento Multimodelo, utilizado nos estudos de Gomes (2011) e Gomes e de Souza (2008), devem ser avaliadas de forma mais detalhada. Os desempenhos mais precisos no procedimento de Emparelhamento Multimodelo podem estar relacionados com a organização visual dos estímulos (três modelos e três comparações simultaneamente disponíveis) e a resposta de arrastar, mas também com a apresentação de pares de estímulos idênticos (estímulos modelo e comparação próximos formam um par de estímulos idênticos). A topografia da resposta requerida no Emparelhamento Multimodelo dos estudos de Gomes (2011) e Gomes e de Souza (2008) envolvia movimentar um estímulo em direção a outro. Assim, a cada resposta mudava a disposição visual do estímulo modelo, que ficava cada vez mais próximos do respectivo estímulo de comparação, até os dois se posicionarem lado-a-lado, antes da resposta de colocar um sobre o outro. O par de estímulos formado dessa maneira pode ser considerado como um estímulo composto com dois elementos iguais. É possível que a proximidade entre os estímulos modelo e de comparação seja uma variável adicional que possa favorecer o controle pela similaridade físi- 
ca, ou igualdade, nas tarefas de emparelhamento de identidade. A partir dessa análise, o par com estímulos idênticos poderia funcionar como consequência para a resposta de arrastar e como estímulo antecedente para a resposta de posicionar (ou soltar) um estímulo sobre o outro.

Os resultados dos estudos previamente descritos (de Freitas, 2012; Gomes, 2011; Shimizu et al., 2003), entretanto, não permitem afirmar qual a característica da tarefa de Emparelhamento Multimodelo é a variável crítica: a resposta de movimentar cada estímulo de comparação até posicioná-lo sobre o respectivo modelo (com resposta de arrastar) ou o estímulo composto com elementos idênticos resultante da aproximação dos estímulos modelo e de comparação. Nos estudos previamente descritos, essas duas variáveis ocorriam de maneira conjunta, uma vez que somente a partir da resposta de arrastar era possível movimentar um estímulo para próximo do outro. No presente estudo, foi proposta como alternativa a utilização de tarefas de emparelhamento com exigência de duas respostas, clicar no estímulo modelo e clicar no estímulo de comparação, para que a consequência de cada pareamento fosse apresentada. Foi comparada uma condição similar a do estudo de Gomes (2011), em que cada pareamento correto na tarefa Multimodelo era sinalizado pela remoção dos estímulos de um dos lados da tela, com uma condição em que era apresentado um par de estímulos idênticos.

O presente estudo é uma replicação parcial do Experimento 1 de Gomes (2011) com participantes com TEA e tarefas informatizadas. O estudo teve como objetivo avaliar o efeito da apresentação de pares de estímulos idênticos como consequência de respostas de pareamento corretas em tarefas de treino de relações de identidade com os procedimentos de emparelhamento Típico e Multimodelo.

\section{Método}

\section{Participantes}

Participaram do estudo 24 indivíduos com TEA, com idades entre cinco a 13 anos, quatro do sexo feminino e 20 do sexo masculino, sendo que quatro não utilizavam a fala para se comunicar. Um participante apresentava perda auditiva progressiva congênita e utilizava aparelho de amplificação sonora individual nos dois ouvidos (Tabela 1). Dezessete participantes eram provenientes de salas de atendimento educacional especializado de uma instituição pública de ensino da cidade de Teresina (PI) e sete crianças eram atendidas em instituição de ensino privada da cidade de Belo Horizonte (MG). Os participantes foram indicados pelos professores a partir dos critérios de seleção que incluíam: diagnóstico prévio de TEA; repertórios básicos para manuseio de computador com a utilização do mouse (e.g., movimentar o cursor com o uso do mouse; posicionar o cursor em locais solicitados e clicar); e ausência de história anterior de participação em pesquisa com o procedimento de emparelhamento ao modelo.

A escala CARS (Childhood Autism Rating Scale - Schopler, Reichler, \& Renner, 1988) foi aplicada com o objetivo de identificar o perfil de habilidades gerais dos participantes. Foram realizadas entrevistas com os pais ou cuidadores, e a pontuação obtida classificada em: Comportamento Apropriado à idade e situação $(15$ - 29,5), autismo Leve/Moderado (30 - 36,5) e autismo Grave (superior a 37).

O estudo foi aprovado por um Comitê de Ética e foi conduzido de acordo com a Resolução do Conselho Nacional de Saúde (CNS) 466/2012, vigente na época de submissão do projeto do presente estudo. Os responsáveis foram informados sobre o estudo e autorizaram a participação dos filhos e a filmagem das sessões por meio da leitura e assinatura de um termo de consentimento livre esclarecido e de outro específico para a utilização de imagem e som de voz.

\section{Local, Equipamentos e Materiais}

A coleta de dados foi realizada em salas disponibilizadas pelas instituições de ensino, equipadas com duas mesas e duas cadeiras. O participante sentava-se na cadeira em frente à mesa com o computador, e o experimentador ficava na cadeira atrás do participante. Na outra mesa eram expostos brinquedos (e.g., carrinhos, bone- 
Tabela 1

Características Gerais dos Participantes: Idade, Sexo, Classificação de Acordo com a CARS, Presença ou não de Repertorio Verbal Vocal e Tipo de Instituição de Ensino/Estado da Federação

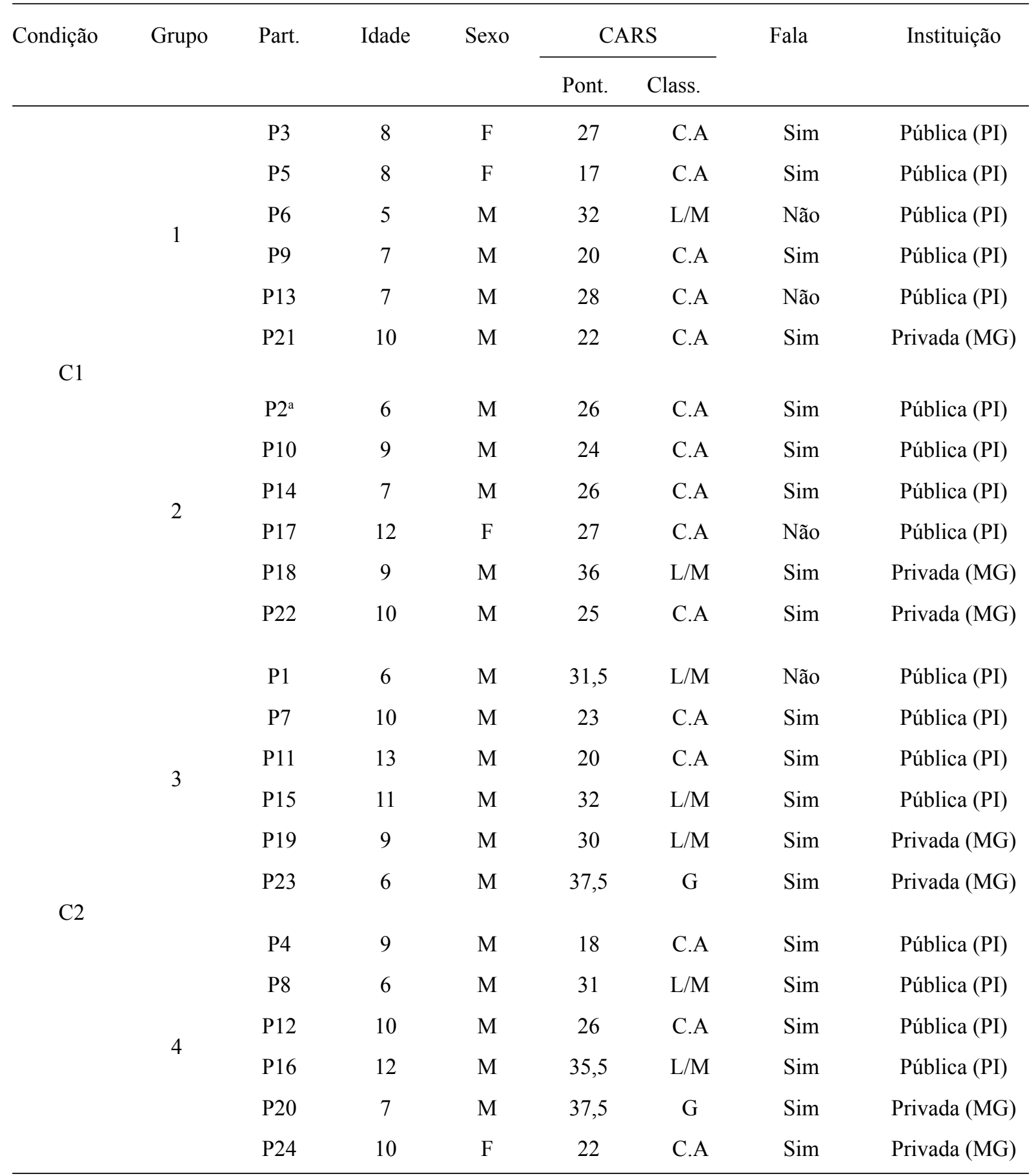

Nota. C.A- Comportamentos Apropriado à situação; L/M- Leve/Moderado; e G- Grave.

${ }^{a}$ Perda auditiva progressiva congênita e utilização de aparelho de amplificação sonora individual nos dois ouvidos.

cos, ursos de pelúcia, bolha de sabão), livros de histórias infantis e vídeos.

O software Contingência Programada versão 2.0 (Hanna, Batitucci, \& Batitucci, 2014), para o sistema Windows, foi utilizado para a programação das tarefas experimentais e registro das respostas de seleção. Para a apresentação dos estímulos foi utilizado um computador portátil Sony Vaio com tela de 10,3", acoplado a um monitor LCD (17") Sony. Para a filmagem das sessões foi utilizada uma câmera digital Sony cyber-shot LCD 2,7", posicionada sobre um tripé. 


\section{Estímulos}

Foram utilizados estímulos visuais de cinco categorias: 24 Símbolos Abstratos (SA), 30 Figuras em Preto e Branco (FPB), 24 Palavras impressas com uma e duas sílabas (P), 15 Sequências Numéricas de quatro dígitos ( $\mathrm{SN})$ e 36 Figuras Coloridas (FC). As figuras foram obtidas no sítio de acesso livre Google Imagens e editadas no programa Paint 6.1. As palavras e as sequências numéricas foram confeccionadas com fonte Times New Roman e tamanho 60. Os estímulos foram apresentados no centro de um retângulo branco com $4 \times 6 \mathrm{~cm}$.

\section{Procedimento Geral}

Os participantes foram divididos em quatro grupos. Os grupos 1 e 2 foram expostos à Condição 1 e os grupos 3 e 4 à Condição 2. Cada Condição Experimental era composta por três blocos de tentativas de tarefas de relações de identidade: Emparelhamento Típico, Emparelhamento Multimodelo, e tentativas misturadas dos dois tipos de tarefa. Um grupo de cada condição foi exposto à sequência de tarefas de emparelhamento Típico-Multimodelo-Misturadas e o outro grupo, à sequência Multimodelo-Típico-Misturadas.

As tarefas de emparelhamento Típico e Multimodelo da Condição 1 eram similares as do estudo de Gomes (2011), sendo que na tarefa Multimodelo um estímulo do lado esquerdo era removido após cada pareamento correto. Entretanto, na Condição 2, após a escolha do estímulo de comparação igual ao modelo, era apresentado na tela da tentativa um estímulo composto, formado por dois elementos iguais, lado-a-lado (par de estímulos idênticos). No Emparelhamento Multimodelo cada estímulo composto era apresentado no local (ou janela) onde estava posicionado o estímulo modelo previamente selecionado. No Emparelhamento Típico esse par de estímulos idênticos era apresentado na tela de reforço de cada pareamento e após o emparelhamento correto de três modelos com as respectivas comparações (três pares simultaneamente).

Os blocos com tentativas de Emparelhamento Típico e de Emparelhamento Multimo- delo eram formados por 10 tentativas de treino e 10 tentativas de teste. O bloco com tentativas misturadas continha apenas tentativas de teste, 10 de cada. Em todas as tentativas de teste eram apresentados estímulos novos, diferentes dos utilizados nas tentativas de treino.

Nas tentativas de treino, para o emparelhamento dos três primeiros modelos com as respectivas comparações, o experimentador apresentava instruções verbais enquanto mostrava a posição dos estímulos e, se necessário, fornecia ajuda física para a resposta de clicar. Foi utilizado como critério de encerramento das tentativas de treino e início das tentativas de teste, três acertos consecutivos em 10 tentativas, sem correção ou ajuda. Caso esse critério não fosse atingido, a sessão era repetida por, no máximo, três vezes. Os participantes que não atingiram o critério dos treinos na terceira exposição foram excluídos do estudo.

Nas tentativas de teste não foram programadas consequências diferenciais para respostas corretas e incorretas. Eventualmente, era apresentado reforço social (e.g., "Você está indo muito bem!"; "Bom trabalho!"; “Ótimo!") para manter o engajamento do participante na tarefa, mas sem relação de contingência com acertos ou erros.

As sessões tinham a duração de 30 a 50 min, dependendo do desempenho do participante e, geralmente, eram acompanhadas por um professor auxiliar, ou estagiário da instituição, que permanecia sentado em um canto da sala e era instruído a intervir somente se fosse solicitado. Dicas verbais (e.g., "Qual figura você vai escolher agora?") eram utilizadas quando os participantes permaneciam por um tempo, de até 15 $\mathrm{s}$, sem emitir uma resposta. No caso de ocorrências de comportamentos disruptivos (e.g., ecolalias, levantar-se da cadeira, estereotipias motoras), a sessão poderia ser interrompida por até $5 \mathrm{~min}$. Nessas ocasiões, o experimentador oferecia um brinquedo ao participante e apresentava instruções tais como "Você precisa sentar nessa cadeira para poder brincar", "Responda essa e depois você poderá brincar". Após 5 min, se não houvesse diminuição na ocorrência de tais comportamentos com esse procedimento 
e/ou com a colaboração do cuidador, a sessão era encerrada.

Ao final da tarefa no computador o participante era convidado a escolher entre brincar com um dos brinquedos ou assistir a algum dos vídeos infantis disponibilizados sobre uma das mesas. Essa atividade tinha a duração máxima de 5 min e era independente da quantidade de acertos nas tarefas realizadas no computador.

A seguir serão caracterizadas as tarefas de Emparelhamento Típico e de Emparelhamento Multimodelo de cada condição experimental.

\section{Condição 1}

Emparelhamento Típico. Em todas as tentativas, era requerida a resposta de clicar sobre o estímulo modelo para a apresentação dos três estímulos de comparação na parte inferior da tela (Figura 2; coluna da esquerda; telas a e b). A tarefa do participante consistia em selecionar o estímulo de comparação idêntico ao modelo. Nas tentativas de treino as consequências para acertos (figura de um smile, sequência de notas musicais - indicada pelo autofalante na tela c, e reforço social por parte do experimentador) e respostas incorretas (" $\mathrm{X}$ " vermelho; tela e) eram apresentadas por 1,5 s. Posteriormente, era apresentada uma tela cinza (tela d) por um período de 1,5 s, denominado de Intervalo Entre Tentativas (IET), e depois por uma nova tentativa. Nas tentativas de teste, as respostas de seleção resultavam apenas na apresentação do IET por 3,0 s.

Emparelhamento Multimodelo. Uma tentativa da tarefa Multimodelo era definida como o emparelhamento correto de três modelos com as respectivas comparações. Para cada conjunto de três estímulos, eram apresentados, inicialmente, três estímulos na coluna à esquerda (modelos) e três estímulos fixos na coluna à direita da tela (comparações). O participante deveria clicar sobre um dos estímulos da coluna da esquerda e, posteriormente, selecionar (clicar) o estímulo igual na coluna da direita. Conforme a Figura 1 (segunda coluna a partir da esquerda), a resposta de clicar sobre um dos estímulos da coluna da esquerda (tela a) produzia a sua remoção e posterior apresentação na janela superior central da tela (tela b). Nas tentativas de treino, respostas corretas resultavam em consequências iguais às da tarefa de emparelhamento típico com o acréscimo de um par de estímulos idênticos (tela c). A apresentação desse estímulo composto sinalizava o emparelhamento correto do modelo com a comparação e foi mantido junto com a tela de reforço para não aumentar a duração da tentativa, caso fosse apresentado separadamente. Cada estímulo corretamente pareado era removido da coluna da esquerda (tela d); a tela "e" ilustra a seleção do terceiro modelo após os dois primeiros emparelhamentos. Após a realização correta dos três emparelhamentos eram apresentados três estímulos compostos na coluna da esquerda (tela f) por 1,5 s. Respostas incorretas resultavam em um "X" vermelho no centro da tela (tela e; primeira coluna à esquerda) por 1,5 s. Não foi utilizado IET após as telas de consequências para respostas corretas, incorretas e de finalização dos três emparelhamentos com o objetivo de tornar a atividade mais dinâmica, o que evitou o aumento da duração da tarefa.

Nas tentativas de teste, cada resposta de emparelhamento (telas a e b) resultava na apresentação do IET por 1,5 s, seguida de uma tela contendo os estímulos ainda não selecionados na coluna da esquerda, de modo similar ao que ocorria nas tentativas de treino (tela d). Ao término do emparelhamento dos três estímulos de cada conjunto era apresentada a tela de IET por 3,0 s.

Tentativas Misturadas de teste de Emparelhamento Típico e de Emparelhamento Multimodelo. As 10 tentativas de Emparelhamento Típico e as 10 de Emparelhamento Multimodelo eram apresentadas de forma randômica e apresentavam as mesmas características das tentativas de teste isolado de Emparelhamento Típico e Multimodelo previamente descritas.

\section{Condição 2}

Emparelhamento Típico. Na Condição 2, as respostas de seleção correta resultavam na apresentação das consequências para acerto da Condição 1 com o acréscimo de um estímulo composto formado por dois elementos iguais (o 
modelo e a comparação previamente pareados). Esse estímulo composto indicava a formação do par (telas a, b e c; terceira coluna da esquerda para a direita). Posteriormente, era apresentada a tela de IET por $1,5 \mathrm{~s}$ e depois uma nova tentativa (tela d). Outra diferença em relação a Condição 1 era que, após a finalização dos três empare- lhamentos, eram apresentados, por $1,5 \mathrm{~s}$, três estímulos compostos na parte inferior da tela, juntamente com a figura do smile, sons de notas musicais e reforço social (tela e). As tentativas de teste apresentavam as mesmas características das tentativas de teste da Condição 1, previamente descritas.

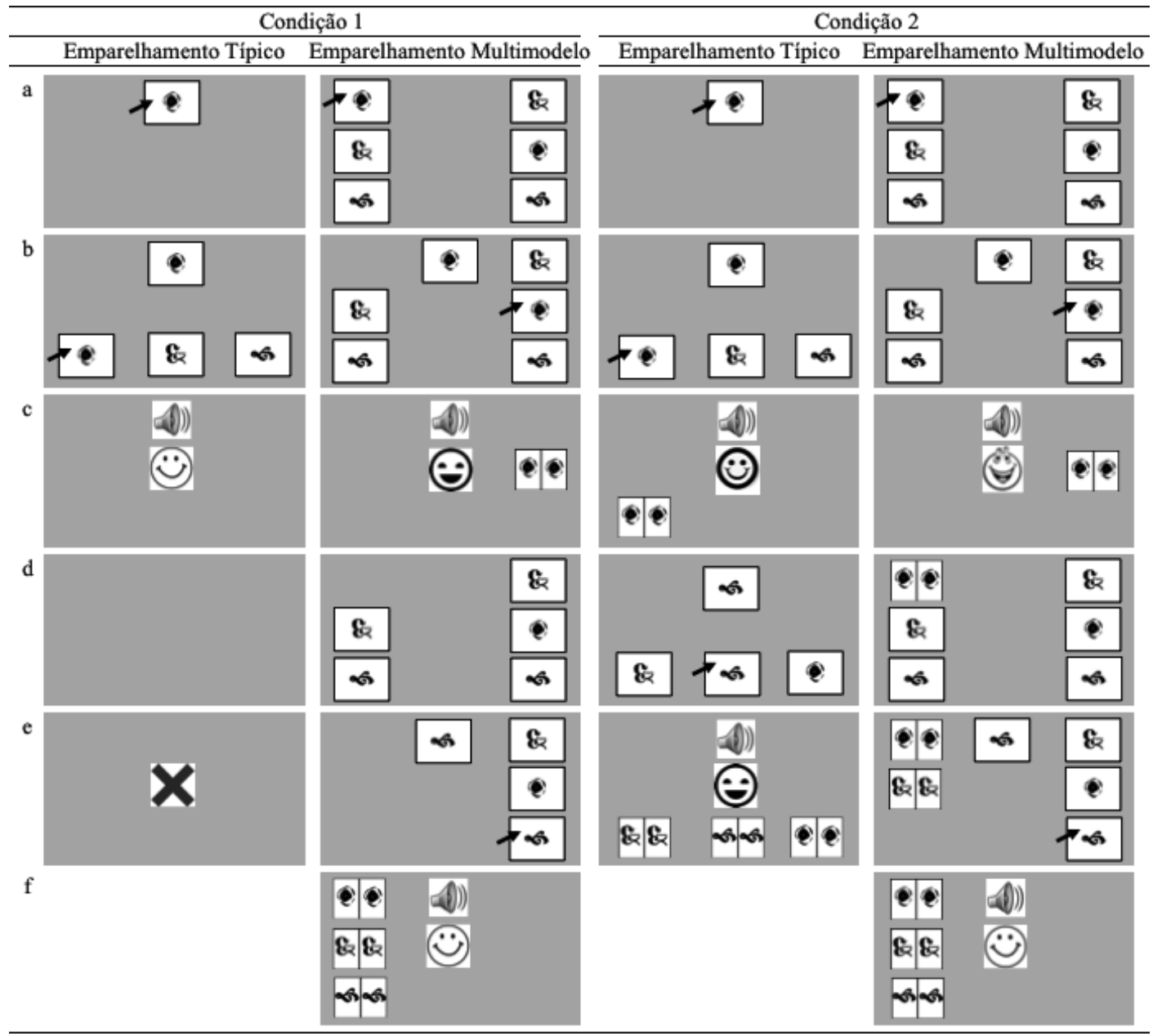

Figura 1. Ilustração de telas das tarefas de Emparelhamento Típico e Emparelhamento Multimodelo da Condição 1 e da Condição 2 (estímulos da categoria Símbolos Abstratos), com a inclusão das consequências para acertos. A consequência para os erros (tela e, primeira coluna a esquerda) e o IET (tela d) eram iguais para todas as condições.

Emparelhamento Multimodelo. Diferentemente da Condição 1, após cada resposta de seleção correta (telas a, b e c), era apresentado um estímulo composto formado por dois elementos iguais (os estímulos pareados: modelo e comparação) na coluna da esquerda, na mesma posição em que era apresentado o estímulo individual antes da resposta de seleção (tela d - um estímulo composto; tela e - dois estímulos compostos). As consequências para o emparelhamento correto dos três estímulos de um mesmo conjunto eram iguais às do treino com Emparelhamento Multimodelo da Condição 1 (tela f) que eram também similares as do Emparelhamento Típico da Condição 2 (tela e). Respostas incorretas resultavam na apresentação de um " $X$ " no centro da tela (tela e; primeira coluna à esquerda), seguida dos estímulos com a mesma disposição visual apresentada antes do erro. 
Nas tentativas de teste, independente de acerto ou erro, após cada resposta de emparelhamento era apresentada a tela de IET 1,5 s, seguida da apresentação, por $1,5 \mathrm{~s}$, de uma tela contendo retângulos em branco no lugar dos estímulos da coluna da esquerda (modelos) previamente selecionados. Tal estratégia foi utilizada para manter a similaridade com as tentativas de treino em que os estímulos pareados eram apresentados lado-a-lado e não removidos como na Condição 1 .

Em todas as tentativas de treino e teste com os procedimentos de Emparelhamento Típico e Multimodelo da Condição 2 os estímulos compostos eram inoperantes, não produziam alteração na tela e as respostas de seleção não eram registradas como acerto ou erro.

Tentativas Misturadas de teste de Emparelhamento Típico e de Emparelhamento Multimodelo. Esse teste foi composto por 10 tentativas de Emparelhamento Típico e 10 tentativas de Emparelhamento Multimodelo da Condição 2, apresentadas de maneira randômica e com as mesmas características das tentativas isoladas de teste de emparelhamento Típico e Multimodelo.

\section{Resultados}

Nas tentativas de treino foi verificada mais exposição ao bloco de Emparelhamento Multimodelo do que ao Emparelhamento Típico nas duas condições, sendo que a quantidade variou de 1-3. Dois participantes (P6 e P13) foram expostos duas vezes aos blocos de treino de Emparelhamento Típico enquanto que seis necessitaram de 2-3 blocos para atingir o critério do treino com Emparelhamento Multimodelo (P1, P6, P8, P13, P18 e P23).

De acordo com análise dos desempenhos individuais (Figura 2), a porcentagem de acerto nas tentativas de teste com Emparelhamento Típico foi maior ou igual a da tarefa de Emparelhamento Multimodelo, independente da ordem de exposição, exceto para P12. Os desempenhos na Condição 2 atingiram escores mais altos do que na Condição 1. Nove participantes apresentaram $100 \%$ de acerto nas tentativas de
Emparelhamento Típico com tentativas isoladas e misturadas, sendo que seis deles também apresentaram escores precisos nas tentativas Multimodelo (P7, P11, P15, P4, P16 e P24). Apenas quatro participantes da Condição 1 (P6, P14, P17 e P18) e dois da Condição 2 (P8 e P20) apresentaram porcentagem de acerto igual ou inferior a $60 \%$ em um ou nos dois tipos de tarefas de emparelhamento.

Como a ordem de exposição aos blocos não afetou o desempenho dos participantes (Figura 2), foi analisada a média da porcentagem de acerto para os 12 participantes dos grupos 1 e 2 e os 12 participantes dos grupos 3 e 4 nas tentativas de teste de emparelhamento Típico e Multimodelo, isoladas e misturadas (Figura 3). A porcentagem média de acerto foi maior na Condição 2 (barras cinza claro) do que na Condição 1 (barras cinza escuro) para os dois tipos de tarefas de emparelhamento (Típico e Multimodelo). Os escores foram maiores para as tentativas de Emparelhamento Típico, tanto apresentadas isoladas (barras à esquerda da linha tracejada vertical) como misturadas (barras à direita) com tentativas de emparelhamento Multimodelo. Para o Emparelhamento Multimodelo, a porcentagem média de acerto nas duas condições foi maior no bloco com tentativas misturadas (as duas últimas barras à direita da linha vertical) em comparação com tentativas isoladas, mas com pouca variação (Condição 1: 70,8 - 75,8; Condição 2: 77,5 - 81,7).

A partir do Teste de Postos Sinalizados de Wilcoxon, um teste não-paramétrico que se destina a comparar duas condições independentes, foi verificado que as diferenças entre as porcentagens média de acerto nas tarefas de Emparelhamento Típico e de Emparelhamento Multimodelo foram significativas $(p<0,05)$ para as duas condições, exceto para as tentativas isoladas da Condição 2, que foi próximo ao nível de significância $(p=0,05)$. 


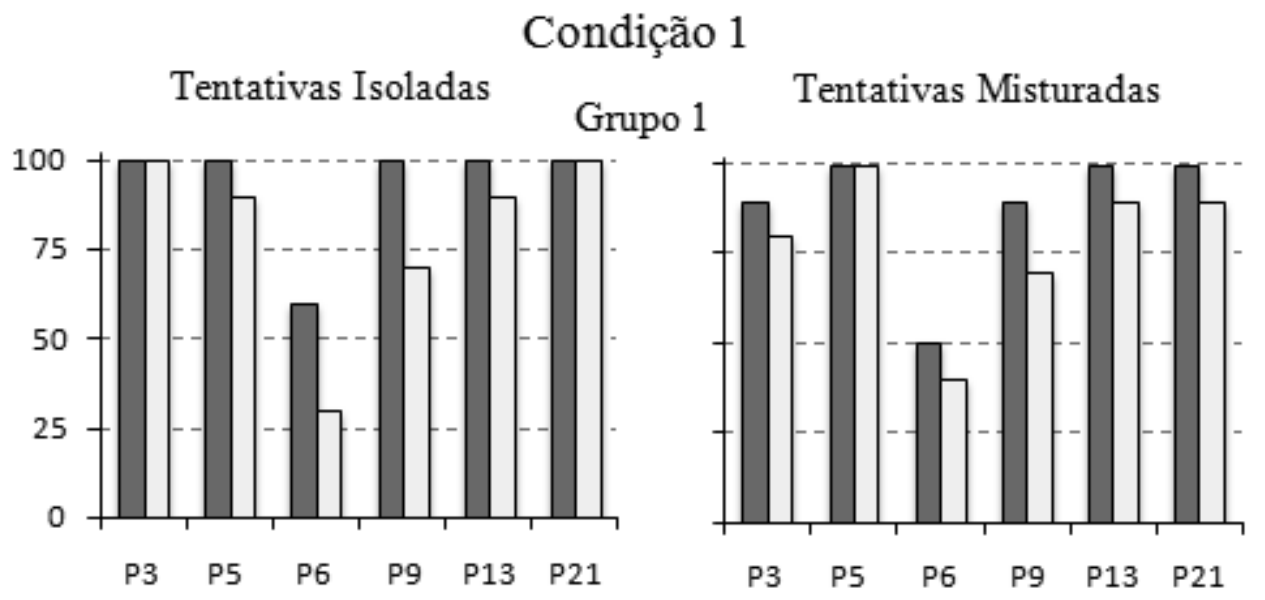

Grupo 2

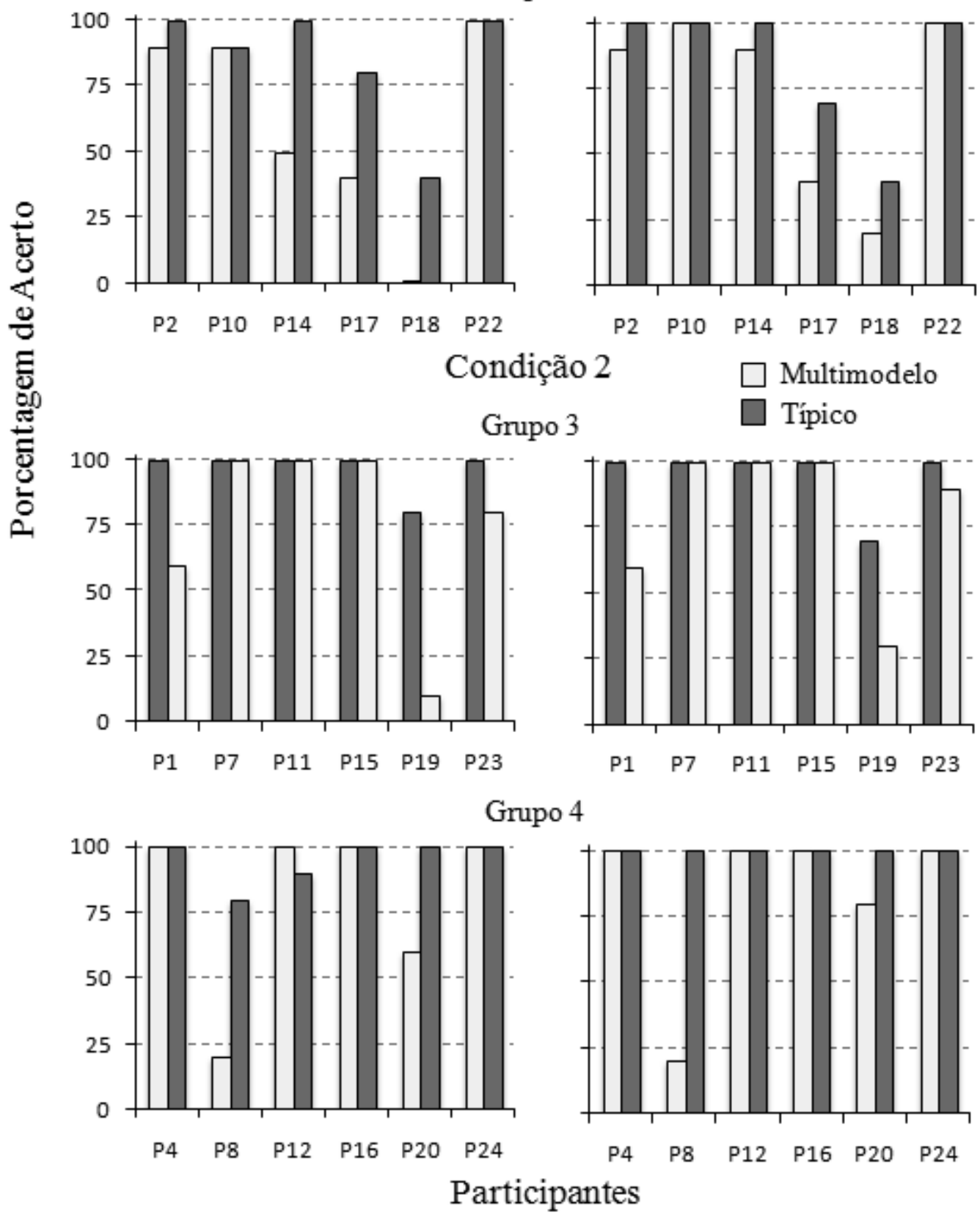

Figura 2. Porcentagem de acerto nas tentativas isoladas e misturadas dos blocos de testes das tarefas de emparelhamento Típico e Multimodelo nas duas condições experimentais. 


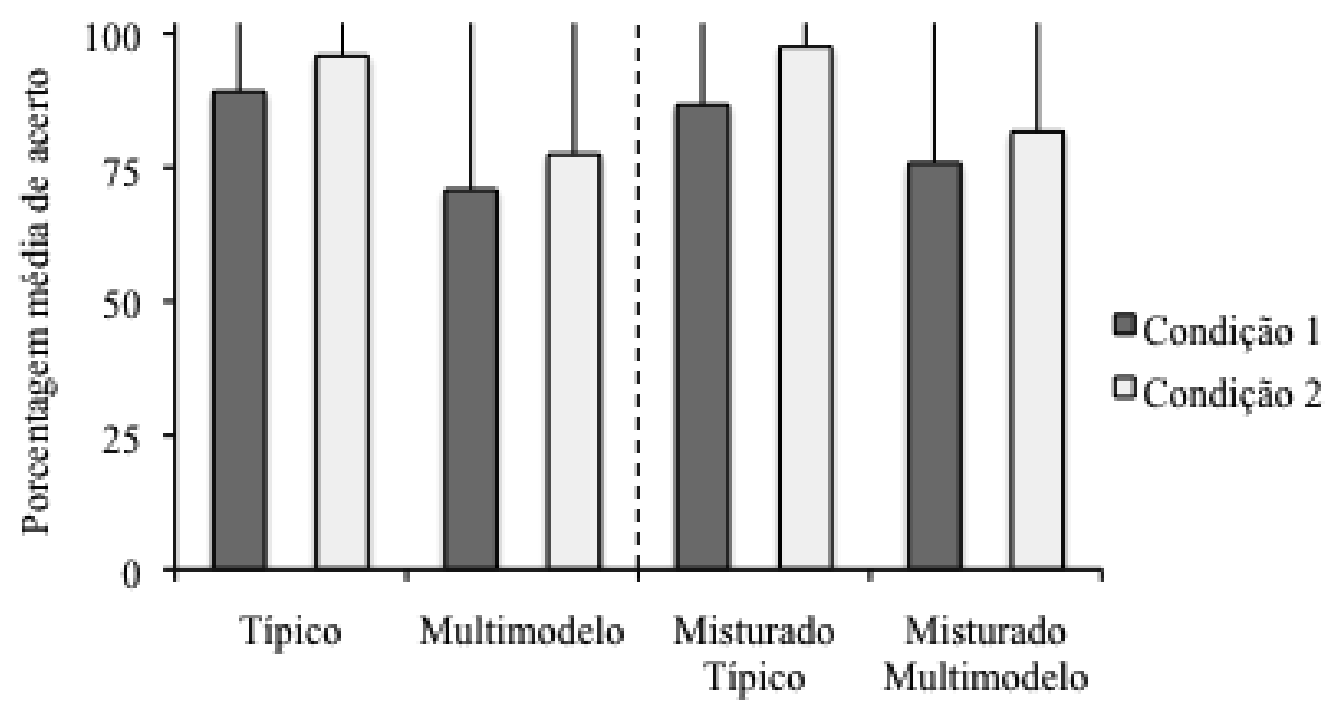

Blocos de tentativas

Figura 3. Porcentagem média de acerto na Condição 1 (grupos 1 e 2) e na Condição 2 (grupos 3 e 4 ) os blocos com tentativas isoladas de teste de emparelhamento Típico e Multimodelo (barras à esquerda da linha tracejada) e no bloco com tentativas misturadas dos dois tipos de tarefas de emparelhamento (barras à direita). A linha vertical contínua indica um desvio padrão.

Quatro participantes, dois da Condição 1 (P6 e P18) e dois da Condição 2 (P8 e P19), apresentaram porcentagem de acerto inferior a $30 \%$ nas tentativas de Emparelhamento Multimodelo, isoladas e misturadas. A partir da observação das filmagens das sessões de cada um dos quatro participantes, foi realizada a categorização das respostas nas tentativas de teste de Emparelhamento Multimodelo (isoladas e misturadas), considerando respostas corretas, incorretas e irrelevantes para a tarefa. As respostas incorretas foram classificadas de acordo com a categoria dos estímulos apresentados na tentativa. No caso das respostas irrelevantes, foram registradas a quantidade de cliques sobre os estímulos inativos: o estímulo que aparecia no centro superior da tela (Centro); os estímulos da coluna da direita antes de clicar em um dos estímulos da coluna da esquerda ou os estímulos da coluna da esquerda (Colunas), imediatamente após uma resposta de seleção de um dos estímulos da coluna da direita (ver Figura 1). Pode-se observar na Tabela 2 que, apesar da quantidade elevada de erros (6-10), nas duas condições experimentais ocorreram mais acertos nas tentativas de Emparelhamento Multimodelo misturadas (variação de 2-4) em relação as tentativas isoladas (variação de 1-3). Os erros foram distribuídos nas cinco categorias dos estímulos apresentados e ocorreu uma quantidade excessiva de respostas irrelevantes para a tarefa nas janelas inativas. Verifica-se uma menor quantidade de respostas na janela central. Para as respostas nas janelas inativas das colunas, observa-se tendência de aumento quando as tentativas eram apresentadas misturadas para três participantes (P18, P8 e P19), sendo que o aumento foi maior para P19.

\section{Discussão}

Independentemente da Condição Experimental, foi verificada porcentagem de acerto igual ou maior nas tentativas de teste de Emparelhamento Típico do que nas tentativas de Emparelhamento Multimodelo, o que não replica os resultados do estudo de Gomes (2011, Experimento 1) em que a maioria dos participantes apresentou melhor desempenho nas tentativas de Emparelhamento Multimodelo.

Nos treinos do presente estudo foram definidos diferentes parâmetros para favorecer o controle de estímulo pela relação de identidade. 
Tabela 2

Quantidade de Acertos e de Erros por Tentativas, de Acordo com Cinco Categorias de Estímulos, e de Respostas Irrelevantes em Janelas Inativas do Centro e das Colunas da Esquerda e Direita, nas Tentativas de Teste de Emparelhamento Multimodelo Isoladas e Misturadas

\begin{tabular}{|c|c|c|c|c|c|c|c|c|c|c|}
\hline & \multirow{3}{*}{ Cond. } & \multirow{3}{*}{ Part. } & \multirow{3}{*}{ Acertos } & \multicolumn{5}{|c|}{ Erros } & \multirow{2}{*}{\multicolumn{2}{|c|}{$\begin{array}{c}\text { Estímulos Inativos } \\
\text { Janelas }\end{array}$}} \\
\hline & & & & \multicolumn{5}{|c|}{ Categorias de Estímulos } & & \\
\hline & & & & FPB & $\mathrm{FC}$ & $\mathrm{SN}$ & $\mathrm{P}$ & SA & Centro & Colunas \\
\hline \multirow{4}{*}{ 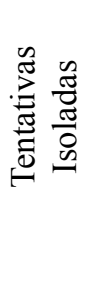 } & 1 & P6 & $3 / 10$ & $1 / 2$ & $2 / 4$ & $1 / 1$ & $1 / 1$ & $2 / 2$ & 20 & 50 \\
\hline & & $\mathrm{P} 18$ & $0 / 10$ & $2 / 2$ & $4 / 4$ & $1 / 1$ & $1 / 1$ & $2 / 2$ & 41 & 45 \\
\hline & 2 & P8 & $2 / 10$ & $1 / 2$ & $3 / 4$ & $1 / 1$ & $1 / 1$ & $2 / 2$ & 31 & 47 \\
\hline & & P19 & $1 / 10$ & $1 / 2$ & $4 / 4$ & $1 / 1$ & $1 / 1$ & $2 / 2$ & 28 & 51 \\
\hline \multirow{4}{*}{ 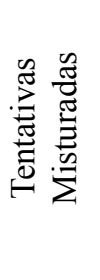 } & 1 & P6 & $4 / 10$ & $1 / 2$ & $1 / 3$ & $1 / 1$ & $1 / 1$ & $2 / 3$ & 15 & 46 \\
\hline & & P18 & $2 / 10$ & $1 / 2$ & $2 / 3$ & $1 / 1$ & $1 / 1$ & $3 / 3$ & 35 & 48 \\
\hline & 2 & P8 & $2 / 10$ & $1 / 2$ & $2 / 3$ & $1 / 1$ & $1 / 1$ & $3 / 3$ & 32 & 50 \\
\hline & & P19 & $3 / 10$ & $1 / 2$ & $2 / 3$ & $1 / 1$ & $1 / 1$ & $2 / 3$ & 16 & 81 \\
\hline
\end{tabular}

Nota. Cond. - Condição; Part. - Participante; FPB - Figuras em Preto e Branco; FC - Figuras Coloridas; SN - Sequências Numéricas; P - Palavras; SA - Símbolos Abstratos; Centro - Respostas de clicar janela inativa do centro; e Colunas - Respostas de Clicar em janelas inativas das colunas da esquerda e da direita.

Foi utilizado como critério de encerramento do treino e início das tentativas de teste, três acertos consecutivos, sem dica ou ajuda do experimentador; e o participante poderia ser exposto até três vezes ao bloco de treino. Diferentemente, no estudo de Gomes (2011; Experimento 1), o critério era de uma tentativa correta, sem correção, em um conjunto de até nove tentativas; e cada participante era exposto uma única vez ao bloco de treino. No presente estudo, a exigência de uma quantidade maior de emparelhamentos reforçados pode ter fortalecido o controle pela relação de identidade. $\mathrm{O}$ efeito desse parâmetro pode ser evidenciado pelos desempenhos próximos ou iguais a $100 \%$, para a maioria dos participantes, nas tentativas de teste de Emparelhamento Típico e de Emparelhamento Multimodelo, nas duas condições (Figura 2).

No estudo de Gomes (2011), tanto no Emparelhamento Típico como no Emparelhamento Multimodelo, era possível mudar, por várias vezes, a resposta ou o estímulo selecionado. A tentativa somente era encerrada quando o participante clicava sobre o botão de finalização (figura de uma mão, no canto inferior direito da tela do computador). Nas tentativas de Emparelhamento Multimodelo, por exemplo, após selecionar e arrastar cada estímulo da coluna da esquerda e colocá-lo sobre um dos estímulos da coluna da direita, o participante poderia modificar o local em que os estímulos foram colocados (comparações sobre os modelos). A organização visual diferenciada da tarefa de Emparelhamento Multimodelo, juntamente com tal possibilidade de mudança de resposta, pode ter maximizado os acertos em comparação com a tarefa de Emparelhamento Típico.

No presente estudo, as tentativas de Emparelhamento Típico finalizavam após cada resposta de seleção de um dos estímulos de comparação e no Emparelhamento Multimodelo, uma tentativa finalizava após três emparelhamentos. Em nenhum dos dois tipos de tarefas havia possibilidade de mudança da resposta de escolha. 
Nas tentativas de treino da tarefa Multimodelo, se o estímulo selecionado da coluna da direita não fosse igual ao da esquerda, era apresentado um "X" no centro da tela, seguida da reapresentação dos estímulos com a mesma disposição visual que precedeu a resposta incorreta (Figura 1). Assim, a utilização de consequência diferencial para cada pareamento permitia, em caso de erro, a ocorrência de uma nova resposta de pareamento. Entretanto, isso não acontecia nas tentativas de teste. Esse procedimento resultou em porcentagem de acerto igual ou maior que $70 \%$ nas tentativas Multimodelo, para a maioria dos participantes, embora tenha sido um pouco mais baixa que no Emparelhamento Típico (ver Figura 2).

Além da forma de apresentação dos estímulos, no estudo de Gomes (2011) a quantidade de respostas requeridas nos dois tipos de emparelhamento era diferente. Nas tentativas de Emparelhamento Típico, os estímulos modelo e de comparação eram apresentados simultaneamente na tela e era requerida uma única resposta, selecionar o estímulo de comparação idêntico ao modelo. Nesse procedimento não era necessária uma resposta de observação, ou seja, tocar ou clicar no estímulo modelo para que as comparações fossem apresentadas (Dube \& McIlvane, 1997, 1999). Por outro lado, na tarefa de Emparelhamento Multimodelo, os três estímulos da esquerda estavam posicionados na horizontal e os da direita na vertical e eram requeridas duas respostas, movimentar um estímulo da esquerda em direção à um estímulo da direita e colocar um estímulo sobre o outro. Tais topografias de resposta no Emparelhamento Multimodelo podem ter favorecido o direcionamento da observação para todos os estímulos, o que Gomes (2011) considerou que aumentou a probabilidade de acerto e o controle pela relação de igualdade entre os estímulos.

Em todas as tarefas do presente estudo o participante deveria emitir duas respostas de clicar para finalizar cada emparelhamento. No Emparelhamento Típico o participante deveria clicar no modelo e em seguida clicar na comparação correspondente. No Emparelhamento Multimo- delo era necessário clicar em um estímulo da coluna da esquerda e, em seguida, no estímulo igual da coluna da direita. A resposta ao estímulo modelo, ou de observação, foi requerida nos dois tipos de tarefas de emparelhamento a fim de garantir contato visual com os estímulos modelo e de comparação. A resposta de observação tem sido utilizada para reduzir controle de estímulo por aspectos irrelevantes da tarefa de emparelhamento ao modelo (e.g., Costa, Schmidt, Dominiconi, \& de Souza, 2013; Dube, Iennaco, \& McIlvane, 1993). É possível que a inclusão da resposta de observação no Emparelhamento Típico tenha sido uma variável relevante para a ocorrência de desempenhos mais precisos nas tentativas de teste de Emparelhamento Típico, verificada no presente estudo em comparação com desempenhos mais baixos relatados no Experimento 1 de Gomes (2011).

Foi observado que os participantes expostos à Condição 1 obtiveram percentual de acerto maior nas tentativas de teste de Emparelhamento Típico do que nas tentativas de Emparelhamento Multimodelo (Figuras 2 e 3), em que cada estímulo modelo, após pareado com um estímulo de comparação, era omitido da tela da tentativa. $\mathrm{Na}$ Condição 2, as tentativas de Emparelhamento Típico diferenciavam-se da Condição 1 , pois ao final da escolha, os estímulos pareados eram apresentados lado-a-lado, formando um estímulo composto com elementos iguais (um par), o que sinalizava o acerto na tela de consequências. Nas tentativas de Emparelhamento Multimodelo, quando os estímulos eram pareados também havia a apresentação do estímulo composto na tela de consequências. Adicionalmente, os pares eram inseridos na coluna da esquerda (tela da tentativa) para sinalizar os acertos e os estímulos já pareados (Figura 1). A apresentação desse estímulo composto pode ter estabelecido, ao longo de uma mesma tentativa, controle sobre a resposta de clicar em outro estímulo da coluna da esquerda. Entretanto, deve-se considerar que esse procedimento não garantia que os participantes direcionassem o olhar para os elementos do estímulo composto. Os resultados sugerem que a apresentação dos estímulos 
compostos favoreceu desempenhos mais precisos nos testes de Emparelhamento Típico e Multimodelo, uma vez que a porcentagem média de acerto na Condição 2 foi maior do que na Condição 1 (Figura 3).

No presente estudo a resposta requerida era clicar em cada estímulo modelo e em seguida no estímulo de comparação idêntico, o que era diferente dos estudos de de Freitas (2012) e Gomes (2011; Experimento 1), que utilizaram resposta de arrastar. Por limitações do software utilizado no presente estudo, os estímulos permaneciam na mesma posição. No entanto, foi programada uma simulação de movimento do estímulo da esquerda para a direita (Figura 1, telas b e "e"). Apesar dessa restrição, as tarefas programadas permitiram avaliar o efeito da organização visual das tarefas de emparelhamento de identidade Típico e Multimodelo e da apresentação de pares de estímulos idênticos, mas em um contexto diferente do estudo de Gomes (2011).

Gomes (2011) e Gomes e de Souza (2008) consideraram que a mudança na quantidade de estímulos apresentados na tela do computador, a cada emparelhamento, favoreceu a identificação do início e do término da tentativa, a partir da remoção de cada estímulo de comparação, do lado esquerdo. Apresentação simultânea dos estímulos modelo e de comparação foi programada para a tarefa Multimodelo da Condição 1 do presente estudo, porém os estímulos eram apresentados em colunas enquanto que no estudo de Gomes (2011) os estímulos de comparações eram organizados na horizontal e os modelos na vertical. Entretanto, os resultados da Condição 1 divergiram dos obtidos por Gomes (2011) e Gomes e de Souza (2008), pois foi demonstrado porcentagem de acerto menor nas tentativas de Emparelhamento Multimodelo do que nas tentativas de Emparelhamento Típico. Deve-se, no entanto, considerar que os vários parâmetros, previamente analisados, que diferenciam o presente estudo dos demais podem ter afetado os resultados obtidos, o que sugere a necessidade de estudos adicionais. Poderia ser investigado se os resultados seriam os mesmos caso não fosse utilizada resposta de observação na tarefa de
Emparelhamento Típico e se fosse utilizada resposta de tocar a tela, como no estudo de Gomes (2011).

Apesar da tarefa Multimodelo do presente estudo ter sido efetiva para a maioria dos participantes, quatro deles (P6, P8, P18 e P19) com autismo leve/moderado (Tabela 1) apresentaram porcentagem de acerto inferior a $30 \%$ e emitiram uma grande quantidade de respostas na presença de estímulos inativos (Tabela 2). É possível que o critério utilizado para as tentativas de treino não tenha sido suficiente para a aprendizagem da sequência de desempenhos necessários para executar a tarefa de Emparelhamento Multimodelo. Os escores baixos indicam que não foi estabelecido controle pela relação de identidade ou que outras dimensões dos estímulos podem ter controlado o desempenho (ver Teoria da Coerência de Topografia de Controle de Estímulos; Dube \& McIlvane, 1996; McIlvane \& Dube, 2003).

Uma contribuição do estudo realizado é a descrição e a análise de diversos parâmetros que devem ser considerados na programação de tarefas de emparelhamento de identidade para indivíduos com TEA, tais como a disposição visual dos estímulos, a utilização de estímulos compostos para indicar pareamentos corretos, a resposta de observação, o critério para mudar da etapa de treino para a de teste, e a apresentação de consequências para a resposta de seleção. A investigação de variáveis de procedimentos que afetam o desempenho em tarefas que envolvem relações entre estímulos, como no presente estudo, pode contribuir para o planejamento de condições de ensino em situações aplicadas, especialmente para indivíduos de classes de educação especial.

\section{Referências}

Albuquerque, A. R., \& Melo, R. M. (2005). Equivalência de estímulos: Conceito, implicações e possibilidades de aplicação. In J. Abreu-Rodrigues \& M. R. Ribeiro (Eds.), Análise do Comportamento: Pesquisa, teoria e aplicação (pp. 244-264). Porto Alegre, RS: Artmed.

Costa, R. A., Schmidt, A., Domeniconi, C., \& de Souza, D. G. (2013). Emparelhamento com o mo- 
delo simultâneo e atrasado: Implicações para a demonstração de equivalência de estímulos por crianças. Temas em Psicologia, 21, 469-482. doi: http://dx.doi.org/10.9788/TP2013.2-13

De Freitas, M. C. (2012). Construção de um programa de ensino de habilidades de pré-requisito de leitura e escrita para pessoas com deficiência mental (Tese de doutorado). Recuperado em http://www.ppgpsi.ufscar.br/defesas/tese-mcf

De Rose, J. C. (1993). Classes de estímulos: Implicações para uma análise comportamental da cognição. Psicologia: Teoria e Pesquisa, 9, 283-303. Recuperado em https://revistaptp.unb.br/index. php/ptp/article/view/1589/545

De Rose, J., \& Bortoloti, R. (2007). A equivalência de estímulos como modelo do significado. Acta Comportamentalia, 15, 83-102. Recuperado em http://www.revistas.unam.mx/index.php/acom/ article/view/14527/13854

De Sousa, D. G., Cortez, M. D., Aggio, N. M., \& de Rose, J. (2012). Aprendizagem relacional e comportamento simbólico no processo de conhecimento do mundo. DI-Revista de Deficiência Intelectual, 3(2), 36-42.

Dube, W. V. (1996). Teaching discrimination skills to persons with mental retardation. In C. Goyos, M. A. Almeida, \& D. G. de Souza (Eds.), Temas em Educação Especial: Vol. 3 (pp. 73-96). São Carlos, SP: Editora da Universidade Federal de São Carlos.

Dube, W. V., Iennaco, F. M., \& McIlvane, W. J. (1993). Generalized identity matching to sample of two-dimensional forms in individuals with intellectual disabilities. Research in Developmental Disabilities, 14, 457-477. doi: https:/doi. org/10.1016/0891-4222(93)90038-L

Dube, W. V., \& McIlvane, W. J. (1996). Some implications of a stimulus control topography analysis for emergent behavior and stimulus classes. In T. R. Zentall \& P. M. Smeets (Eds.), Stimulus class formation in humans and animals (pp. 197-218). Amsterdan: Elsevier.

Dube, W. V., \& McIlvane, W. J. (1997). Reinforcer frequency and restricted stimulus control. Journal of Experimental Analysis of Behavior, 68, 303-316. doi: 10.1901/jeab.1997.68-303

Dube W. V., \& McIlvane, W. J. (1999). Reduction of stimulus overselectivity with nonverbal differential observing responses. Journal of Applied
Behavior Analysis, 32, 25-33. doi: 10.1901/ jaba.1999.32-25

Eikeseth, S., \& Smith, T. (1992). The developmental of function and equivalence classes in highfunctioning autistic children: The role of naming. Journal of the Experimental Analysis of Behavior, 58, 123-133. doi: 10.1901/jeab.1992.58-123

Gomes, C. G. (2011). Aprendizagem relacional, comportamento simbólico e ensino de leitura a pessoas com Transtornos do Espectro do Autismo (Tese de doutorado). Recuperado em https://repositorio.ufscar.br/handle/ufscar/2903

Gomes, C. G., \& de Souza, D. G. (2008). Desempenho de pessoas com autismo em tarefas de emparelhamento com o modelo por identidade: Efeito da organização dos estímulos. Psicologia Reflexão e Crítica, 21, 418-429. doi: http://dx.doi. org/10.1590/S0102-79722008000300010

Hanna, E. S., Batitucci, L. A. V., \& Batitucci, J. S. L. (2014). Software Contingência Programada: Utilidade e funcionalidades. Revista Brasileira de Análise do Comportamento, 10, 97-104. doi: http://dx.doi.org/10.18542/rebac.v10i1.3949

Kelly, S., Green, G., \& Sidman, M. (1998). Visual identity matching and auditory visual matching: A procedural note. Journal of Applied Behavior Analysis, 31, 237-243. doi: 10.1901/ jaba.1998.31-237

McIlvane, W. J, \& Dube, W. V. (2003). Stimulus control topography coherence theory: Foundations and extensions. The Behavior Analyst, 26, 195-213. Retrieved from https://www.ncbi.nlm. nih.gov/pmc/articles/PMC2731455/pdf/behavan00006-0017.pdf

Mesibov, G. B., Schopler, E., \& Hearsey, A. (1994). Structured teaching. In E. Schopler \& G. B. Mesibov (Eds.), Behavior issues in autism (pp. 193205). New York: Plenum Press.

Schopler, E., Reichler, J. R., \& Renner, C. (1988). CARS - The Childhood Autism Rating Scale. Los Angeles, CA: Westerm Pychological Services.

Shimizu, H., Twyman, J. S., \& Yamamoto, J. (2003). Computer-based sorting-to-matching in identity matching for young children with developmental disabilities. Research in Developmental Disabilities, 24, 183-194. doi: http://dx.doi.org. ez54.periodicos.capes.gov.br/10.1016/S08914222(03)00028-3 
Smith, T. (2001). Discrete trial training in the treatment of autism. Focus on Autism and Other Developmental Disabilities, 16, 86-92. doi: https:// doi.org/10.1177/108835760101600204

Stromer, R., \& Stromer, J. B. (1989). Children's identity matching and oddity: Assessing control by specific and general sample-comparison relations. Journal of the Experimental Analysis of Behavior, 51, 47-64. doi: 10.1901/ jeab.1989.51-47
Williams, G., Pérez-González, L. A., \& Queiroz, A. (2005). Using a combined blocking procedure to teach color discrimination to a child with autism. Journal of Applied Behavior Analysis, 38, 555558. doi: 10.1901/jaba.2005.65-04

Recebido: 03/01/2017

$1^{a}$ revisão: 09/04/2017 Aceite final: 23/04/2017 